\title{
Relation of parameters of noncommutative algebra with mass and the equivalence principle in a space with quadratic noncommutativity of coordinates
}

\author{
Kh. P. Gnatenko, O. I. Hryhorchak \\ Ivan Franko National University of Lviv, Department for Theoretical \\ Physics \\ 12 Drahomanov St., Lviv, 79005, Ukraine \\ e-mail: khrystyna.gnatenko@gmail.com
}

We find that relation of parameters of noncommutative algebra with mass gives a possibility to solve important problems in quadratic space-time. We show that if parameter of noncommutative algebra is proportional to mass the coordinates in quadratic space-time can be considered as kinematic variables, also the weak equivalence principle is recovered and relations for coordinates and momenta of the center-of-mass of a body correspond to relations of noncommutative algebra with effective parameter.

Ключові слова: quadratic noncommutativity, equivalence principle, kinematic variables

\section{Introduction}

Studies of different physical problems in quantum space have received much attention recently. This interest is motivated by the development of the String Theory and the Quantum Gravity (see, for instance [1,2])

A space with canonical noncommutativity of coordinates is characterized by the following relations

$$
\left[X_{\mu}, X_{\nu}\right]=i \hbar \theta_{\mu \nu}
$$

where $\theta_{\mu \nu}$ are elements of antisymmetric matrix which are constants. Commutation relations for coordinates in a space with Lie-algebraic noncommutativity reads

$$
\left[X_{\mu}, X_{\nu}\right]=i \hbar \theta_{\mu \nu}^{\rho} X_{\rho}
$$

A space with quadratic deformation is characterized by the following relations

$$
\left[X_{\mu}, X_{\nu}\right]=i \hbar \theta_{\mu \nu}^{\rho \tau} X_{\rho} X_{\tau}
$$

(c) Kh. P. Gnatenko, O. I. Hryhorchak, 2019 
In (2), (3) parameters $\theta_{\mu \nu}^{\rho}, \theta_{\mu \nu}^{\rho \tau}$ are particularly chosen constants, which are antisymmetric to lover indexes.

Noncommutative phase space of canonical type is well studied. In the frame of the space many different problems were considered. Among them, for instance, are free particles [3-5], classical systems with various potentials [6-8] and many others. Many papers are devoted also to studies of the coordinate dependent noncommutativity (see, for example, [9-15]).

In general case parameters of noncommutativity $\theta_{\mu \nu}, \theta_{\mu \nu}^{\rho}, \theta_{\mu \nu}^{\rho \tau}$ can be considered to be different for different particles. So, one faces the problem of description of the motion of the center-of-mass of a body in quantum space. The problems of many particles were examined in the frame of noncommutative space of canonical type $[3,16,17]$, in noncommutative space-time [18], in twisted N-enlarged Newton-Hooke space-time [19]. In papers $[20,21]$ it was shown that to solve the problem of description of motion of composite system in noncommutative space it is important to consider parameters of noncommutativity to be related with mass. Besides this relation opens possibility to recover the weak equivalence principle, to preserve properties of the kinetic energy, to solve the problem of kinematic variables in noncommutative space of canonical type [20-23]. Note also that idea to relate parameters of deformed algebra with mass opens possibility to obtain important results also in deformed space with minimal length [24,25].

In the present paper we find that the idea to relate parameters of noncommutativity with mass opens possibility to solve a list of problems in a space with quadratic deformation. Namely we find that in a space with quadratic noncommutativity of coordinates relations for coordinates and momenta of the center-of-mass of a composite system reproduce relations of noncommutative algebra and the weak equivalence principle is recovered, coordinates can be considered as kinematic variables, if parameters of noncommutativity are related with mass.

The paper is organized as follows. Section 2 is devoted to studies of the problem of kinematic variables in a space with quadratic noncommutativity of coordinates. Features of description of composite system motion are examined in Section 3. In Section 4 the weak equivalence principle is studied in a space with quadratic noncommutativity and condition for recovering of this principle is proposed. Conclusions are presented in Section 5 .

\section{Kinematic variables in quadratic space-time}

Let us consider a space with quadratic noncommutativity which is characterized by the following relations

$$
\begin{array}{r}
{\left[X_{k}, X_{\gamma}\right]=\frac{i \hbar}{\bar{\kappa}} t X_{l}, \quad\left[X_{l}, X_{\gamma}\right]=-\frac{i \hbar}{\bar{\kappa}} t X_{k},} \\
{\left[X_{k}, X_{l}\right]=0, \quad\left[P_{n}, P_{m}\right]=0,} \\
{\left[X_{\gamma}, P_{k}\right]=-\frac{i \hbar}{\bar{\kappa}} t P_{l}, \quad\left[X_{\gamma}, P_{l}\right]=\frac{i \hbar}{\bar{\kappa}} t P_{k},} \\
{\left[X_{i}, P_{j}\right]=\delta_{i j}, \quad\left[X_{\gamma} P_{\gamma}\right]=1, \quad\left[P_{n}, P_{m}\right]=0,}
\end{array}
$$


here indexes $k, l, \gamma$ are fixed, $k \neq l \neq \gamma$, also $i \neq \gamma, j \neq \gamma$ and $n, m=(1,2,3)[26]$.

In the classical limit $\hbar \rightarrow 0$ from (4)-(7) one obtains the corresponding Poisson brackets

$$
\begin{array}{r}
\left\{X_{k}, X_{\gamma}\right\}=\frac{1}{\bar{\kappa}} t X_{l}, \quad\left\{X_{l}, X_{\gamma}\right\}=-\frac{1}{\bar{\kappa}} t X_{k}, \\
\left\{X_{k}, X_{l}\right\}=0, \quad\left\{P_{n}, P_{m}\right\}=0, \\
\left\{X_{\gamma}, P_{k}\right\}=-\frac{1}{\bar{\kappa}} t P_{l}, \quad\left\{X_{\gamma}, P_{l}\right\}=\frac{1}{\bar{\kappa}} t P_{k}, \\
\left\{X_{i}, P_{j}\right\}=\delta_{i j}, \quad\left\{X_{\gamma} P_{\gamma}\right\}=1, \quad\left\{P_{n}, P_{m}\right\}=0 .
\end{array}
$$

Coordinates and momenta which satisfy (8)-(11) can be represented as

$$
\begin{array}{r}
X_{\gamma}=x_{\gamma}+\frac{1}{\bar{\kappa}} t x_{l} p_{k}-\frac{1}{\bar{\kappa}} t x_{k} p_{l}, \\
X_{k}=x_{k}, \quad X_{l}=x_{l}, \\
P_{n}=p_{n} .
\end{array}
$$

here coordinates $x_{n}$ and momenta $p_{n}$ satisfy the ordinary relations

$$
\begin{array}{r}
\left\{x_{n}, x_{m}\right\}=0, \\
\left\{x_{n}, p_{m}\right\}=\delta_{n m}, \\
\left\{p_{n}, p_{m}\right\}=0 .
\end{array}
$$

Note that coordinate $X_{\gamma}$ depends on the momenta therefore it depends on mass and cannot be considered as kinematic variable in the quadratic space-time. It is important to mention that if parameter $\bar{\kappa}$ of algebra (8)-(11) is related with mass $m$ as

$$
\bar{\kappa}=m \gamma_{\bar{\kappa}}
$$

where $\gamma_{\bar{\kappa}}$ are constants which are the same for different particles, one has

$$
X_{\gamma}=x_{\gamma}+t x_{l} \frac{p_{k}}{m \gamma_{\bar{\kappa}}}-t x_{k} \frac{p_{l}}{m \gamma_{\bar{\kappa}}} .
$$

In (19) one has terms with $p_{k} / m$ and $p_{l} / m$. So, coordinate $X_{\gamma}$ does not depend on mass. Therefore, due to condition (18) the coordinates in quadratic space-time can be considered as kinematic variables.

In the next section we show that the same condition (18) is also important for recovering of relations of noncommutative algebra for coordinates and momenta of the centerof-mass of a composite system.

\section{Relations of noncommutative algebra for coordinates and momenta of the center-of-mass}

Let us consider composite system made of $N$ particles with masses $m_{a}$ in quadratic space time. Coordinates and momenta of particles satisfy relations of algebra (8)-(11) 
with parameters $\bar{\kappa}^{(a)}$

$$
\begin{array}{r}
\left\{X_{k}^{(a)}, X_{\gamma}^{(b)}\right\}=\frac{\delta_{a b}}{\bar{\kappa}^{(a)}} t X_{l}^{(a)}\left\{X_{l}^{(a)}, X_{\gamma}^{(b)}\right\}=-\frac{\delta_{a b}}{\bar{\kappa}^{(a)}} t X_{k}^{(a)}, \\
\left\{X_{k}^{(a)}, X_{l}^{(b)}\right\}=0, \quad\left\{P_{n}^{(a)}, P_{m}^{(b)}\right\}=0, \\
\left\{X_{\gamma}^{(a)}, P_{k}^{(b)}\right\}=-\frac{\delta_{a b}}{\bar{\kappa}^{(a)}} t P_{l}^{(a)}, \quad\left\{X_{\gamma}^{(a)}, P_{l}^{(b)}\right\}=\frac{\delta_{a b}}{\bar{\kappa}^{(a)}} t P_{k}^{(a)}, \\
\left\{X_{i}^{(a)}, P_{j}^{(b)}\right\}=\delta_{i j} \delta_{a b}, \quad\left\{X_{\gamma}^{(a)} P_{\gamma}^{(b)}\right\}=\delta_{a b}, \\
\left\{P_{n}^{(a)}, P_{m}^{(b)}\right\}=0 .
\end{array}
$$

here indexes $a, b$ label the particles.

Defining coordinates and momenta of the center-of-mass of composite system in traditional way $\tilde{\mathbf{P}}=\sum_{a} \mathbf{P}^{(a)}, \tilde{\mathbf{X}}=\sum_{a} \mu_{a} \mathbf{X}^{(a)}$ with $\mu_{a}=m_{a} / \sum_{a} m_{a}$, and taking into account that coordinates and momenta of particles satisfy relations (20)-(24), one finds that the Poisson brackets for coordinates and momenta of the center-of-mass read

$$
\begin{array}{r}
\left\{\tilde{X}_{k}, \tilde{X}_{\gamma}\right\}=\sum_{a} \mu_{a}^{2} \frac{1}{\bar{\kappa}^{(a)}} t X_{l}^{(a)} \\
\left\{\tilde{X}_{l}, \tilde{X}_{\gamma}\right\}=-\sum_{a} \mu_{a}^{2} \frac{1}{\bar{\kappa}^{(a)}} t X_{k}^{(a)}, \\
\left\{\tilde{X}_{k}, \tilde{X}_{l}\right\}=0, \quad\left\{\tilde{P}_{n}, \tilde{P}_{m}\right\}=0, \\
\left\{\tilde{X}_{\gamma}, \tilde{P}_{k}\right\}=-\sum_{a} \mu_{a} \frac{1}{\bar{\kappa}^{(a)}} t P_{l}^{(a)}, \\
\left\{\tilde{X}^{(a)}, \tilde{P}_{l}\right\}=\sum_{a} \mu_{a} \frac{1}{\bar{\kappa}^{(a)}} t P_{k}^{(a)}, \\
\left\{\tilde{X}_{i}, \tilde{P}_{j}\right\}=\delta_{i j}, \quad\left\{\tilde{X}_{\gamma}, \tilde{P}_{\gamma}\right\}=1, \quad\left\{\tilde{P}_{n}, \tilde{P}_{m}\right\}=0 .
\end{array}
$$

Note that relations (25)-(30) do not reproduce relations of noncommutative algebra for coordinates and momenta of individual particles (20)-(24). In the right-hand side of relations $(25),(26),(28),(29)$ one does not have coordinates or momenta of the centerof-mass.

It is important to mention that if parameter $\bar{\kappa}_{a}$ of noncommutative algebra (20)-(24) is proportional to mass, namely if condition(18) is satisfied one has

$$
\begin{aligned}
& \left\{\tilde{X}_{k}, \tilde{X}_{\gamma}\right\}=\frac{1}{\bar{\kappa}_{e f f}} t \tilde{X}_{l}, \quad\left\{\tilde{X}_{l}, \tilde{X}_{\gamma}\right\}=-\frac{1}{\bar{\kappa}_{e f f}} t \tilde{X}_{k}, \\
& \left\{\tilde{X}_{\gamma}, \tilde{P}_{k}\right\}=-\frac{1}{\bar{\kappa}_{e f f}} t \tilde{P}_{l}, \quad\left\{\tilde{X}^{(a)}, \tilde{P}_{l}\right\}=\frac{1}{\bar{\kappa}_{e f f}} t \tilde{P}_{k} .
\end{aligned}
$$

Effective parameter $\bar{\kappa}_{e f f}$ is defined as

$$
\bar{\kappa}_{e f f}=\gamma_{\bar{\kappa}} M,
$$


where $M$ is the total mass of the system $M=\sum_{a} m_{a}$. So, in the case when parameter $\bar{\kappa}_{a}$ is proportional to mass $m_{a}$ coordinates and momenta of the center-of-mass satisfy noncommutative algebra with effective parameter $\bar{\kappa}_{e f f}$. Note that similarly as parameters $\bar{\kappa}_{a}$ which corresponds to a particle and are proportional to its mass (18), effective parameter $\bar{\kappa}_{e f f}$ describing the motion of the center-of-mass of a system is proportional to the total mass of the system. So, condition (18) can be generalized as

$$
\frac{\bar{\kappa}_{a}}{m_{a}}=\frac{\bar{\kappa}_{e f f}}{M}=\gamma_{\bar{\kappa}}
$$

We would like to stress that in quadratic space-time the motion of the center-of-mass is not independent of the relative motion because of relations

$$
\begin{array}{r}
\left\{\Delta X_{k}^{(a)}, \tilde{X}_{\gamma}\right\}=\left\{\tilde{X}_{k}, \Delta X_{\gamma}^{(a)}\right\}=\frac{\mu_{a}}{\bar{\kappa}_{a}} t X_{l}^{(a)}-\sum_{b} \frac{\mu_{b}^{2}}{\bar{\kappa}_{b}} t X_{l}^{(b)}, \\
\left\{\Delta X_{l}^{(a)}, \tilde{X}_{\gamma}\right\}=\left\{\tilde{X}_{l}, \Delta X_{\gamma}^{(a)}\right\}=-\frac{\mu_{a}}{\tilde{\kappa}_{a}} t X_{k}^{(a)}+\sum_{b} \frac{\mu_{b}^{2}}{\tilde{\kappa}_{b}} t X_{k}^{(b)}, \\
\left\{\tilde{P}_{k}, \Delta X_{\gamma}^{(a)}\right\}=\frac{1}{\bar{\kappa}_{a}} t P_{l}^{(a)}-\sum_{b} \frac{\mu_{b}}{\bar{\kappa}_{b}} t P_{l}^{(b)}, \\
\left\{\tilde{P}_{l}, \Delta X_{\gamma}^{(a)}\right\}=-\frac{1}{\bar{\kappa}_{a}} t P_{k}^{(a)}+\sum_{b} \frac{\mu_{b}}{\bar{\kappa}_{b}} t P_{k}^{(b)}, \\
\left\{\Delta P_{k}^{(a)}, \tilde{X}_{\gamma}\right\}=\frac{\mu_{a}}{\bar{\kappa}_{a}} t P_{l}^{(a)}-\sum_{b} \frac{\mu_{a} \mu_{b}}{\tilde{\kappa}_{b}} t P_{l}^{(b)}, \\
\left\{\Delta P_{l}^{(a)}, \tilde{X}_{\gamma}\right\}=-\frac{\mu_{a}}{\bar{\kappa}_{a}} t P_{k}^{(a)}+\sum_{b} \frac{\mu_{a} \mu_{b}}{\tilde{\kappa}_{b}} t P_{k}^{(b)},
\end{array}
$$

and $\left\{\Delta X_{k}^{(a)}, \tilde{X}_{l}\right\}=\left\{\Delta X_{l}^{(a)}, \tilde{X}_{k}\right\}=0,\left\{\tilde{P}_{k}, \Delta X_{l}^{(a)}\right\}=\left\{\tilde{P}_{l}, \Delta X_{k}^{(a)}\right\}=0,\left\{\Delta P_{l}^{(a)}, \tilde{X}_{k}\right\}=$ $\left\{\Delta P_{k}^{(a)}, \tilde{X}_{l}\right\}=0$. Here $\Delta \mathbf{X}^{a}, \Delta \mathbf{P}^{a}$ are coordinates and momenta of the relative motion defined in the traditional way $\Delta \mathbf{P}^{a}=\mathbf{P}^{(a)}-\mu_{a} \tilde{\mathbf{P}}, \Delta \mathbf{X}^{(a)}=\mathbf{X}^{(a)}-\tilde{\mathbf{X}}$. Note that if condition (34) holds these relations are simplified. And one has

$$
\begin{array}{r}
\left\{\Delta X_{k}^{(a)}, \tilde{X}_{\gamma}\right\}=\left\{\tilde{X}_{k}, \Delta X_{\gamma}^{(a)}\right\}=\frac{1}{\bar{\kappa}_{e f f}} t \Delta X_{l}^{(a)}, \\
\left\{\Delta X_{l}^{(a)}, \tilde{X}_{\gamma}\right\}=\left\{\tilde{X}_{l}, \Delta X_{\gamma}^{(a)}\right\}=-\frac{1}{\bar{\kappa}_{e f f}} t \Delta X_{k}^{(a)}, \\
\left\{\tilde{P}_{k}, \Delta X_{\gamma}^{(a)}\right\}=\frac{1}{\bar{\kappa}_{a}} t \Delta P_{l}^{(a)}, \\
\left\{\tilde{P}_{l}, \Delta X_{\gamma}^{(a)}\right\}=-\frac{1}{\bar{\kappa}_{a}} t \Delta P_{k}^{(a)},
\end{array}
$$




$$
\begin{gathered}
\left\{\Delta P_{k}^{(a)}, \tilde{X}_{\gamma}\right\}=\frac{1}{\bar{\kappa}_{e f f}} t \Delta P_{l}^{(a)}, \\
\left\{\Delta P_{l}^{(a)}, \tilde{X}_{\gamma}\right\}=-\frac{1}{\bar{\kappa}_{e f f}} t \Delta P_{k}^{(a)},
\end{gathered}
$$

In the next section we show that condition (34) is also important for recovering of the weak equivalence principle in a space with quadratic noncommutativity.

\section{Equivalence principle in quadratic space-time}

Let us study a particle of mass $m$ in gravitational field $V(\mathbf{X})$ in the space with quadratic noncommutativity (8)-(11). The Hamiltonian of the particle reads

$$
H=\frac{P^{2}}{2 m}+m V(\mathbf{X}) \text {. }
$$

Coordinates and momenta in (47) satisfy relations (8)-(11). So, taking into account (8)(11) one has the following equations of motion

$$
\begin{array}{r}
\dot{X}_{k}=\frac{P_{k}}{m}+m t X_{l} \frac{1}{\bar{\kappa}} \frac{\partial V(\mathbf{X})}{\partial X_{\gamma}}, \\
\dot{X}_{l}=\frac{P_{l}}{m}-m t X_{k} \frac{1}{\bar{\kappa}} \frac{\partial V(\mathbf{X})}{\partial X_{\gamma}}, \\
\dot{X}_{\gamma}=\frac{P_{\gamma}}{m}-m t X_{l} \frac{1}{\bar{\kappa}} \frac{\partial V(\mathbf{X})}{\partial X_{k}}+m t X_{k} \frac{1}{\bar{\kappa}} \frac{\partial V(\mathbf{X})}{\partial X_{l}}, \\
\dot{P}_{k}=-m \frac{\partial V(\mathbf{X})}{\partial X_{k}}+m t P_{l} \frac{1}{\bar{\kappa}} \frac{\partial V(\mathbf{X})}{\partial X_{\gamma}}, \\
\dot{P}_{l}=-m \frac{\partial V(\mathbf{X})}{\partial X_{l}}-m t P_{k} \frac{1}{\bar{\kappa}} \frac{\partial V(\mathbf{X})}{\partial X_{\gamma}}, \\
\dot{P}_{\gamma}=-m \frac{\partial V(\mathbf{X})}{\partial X_{\gamma}} .
\end{array}
$$

In general case of arbitrary field the equations of motion of a particle in quadratic spacetime were written in [26]. We would like to stress that because of relations (8)-(11) one has that the equations of motion of a particle in gravitational field depend on its mass. Therefore we can conclude that the weak equivalence principle does not hold in the frame of relations (8)-(11).

Note that considering relation (18), one can write

$$
\begin{aligned}
& \dot{X}_{k}=v_{k}+t X_{l} \frac{1}{\gamma_{\bar{\kappa}}} \frac{\partial V(\mathbf{X})}{\partial X_{\gamma}}, \\
& \dot{X}_{l}=v_{l}-t X_{k} \frac{1}{\gamma_{\bar{\kappa}}} \frac{\partial V(\mathbf{X})}{\partial X_{\gamma}},
\end{aligned}
$$




$$
\begin{array}{r}
\dot{X}_{\gamma}=v_{\gamma}-t X_{l} \frac{1}{\gamma \bar{\kappa}} \frac{\partial V(\mathbf{X})}{\partial X_{k}}+t X_{k} \frac{1}{\gamma_{\bar{\kappa}}} \frac{\partial V(\mathbf{X})}{\partial X_{l}}, \\
\dot{v}_{k}=-\frac{\partial V(\mathbf{X})}{\partial X_{k}}+t v_{l} \frac{1}{\gamma_{\bar{\kappa}}} \frac{\partial V(\mathbf{X})}{\partial X_{\gamma}} \\
\dot{v}_{l}=-m \frac{\partial V(\mathbf{X})}{\partial X_{l}}-t v_{k} \frac{1}{\gamma_{\bar{\kappa}}} \frac{\partial V(\mathbf{X})}{\partial X_{\gamma}} \\
\dot{v}_{\gamma}=-\frac{\partial V(\mathbf{X})}{\partial X_{\gamma}}
\end{array}
$$

where

$$
v=\frac{\mathbf{P}}{m}
$$

We have that if condition (18) holds the equations of motion (54)-(59) do not depend on the mass. From this follows that their solutions $\mathbf{X}(t), \boldsymbol{v}(t)$ do not depend on the mass and the trajectory in gravitational field in quadratic space-time is the same for particles with different masses. So, the weak equivalence principle can be recovered in quadratic space-time due to condition (18).

In the case of motion of a macroscopic body of mass $M$ in the quadratic space-time, considering Hamiltonian

$$
H=\frac{\tilde{P}^{2}}{2 M}+M V(\tilde{\mathbf{X}}) .
$$

and taking into account (31)-(32), (33) one obtains equations (54)-(59) for $\tilde{X}_{i}$ and $\tilde{v}_{i}=$ $\tilde{X}_{i} / M$. Note, that writing $(61)$, we assume that the influence of the relative motion on the motion of the center-of-mass of a body can be neglected. So, if relation (34) hold one has that the motion of the center-of-mass of macroscopic body in gravitational field does not depend on its mass and composition. Therefore, the weak equivalence principle is satisfied.

\section{Conclusions}

We have considered a space with quadratic noncommutativity of coordinates characterized by relations (8)-(11). The problem of kinematic variables has been studied in the space. We have shown that noncommutative coordinates depend on mass, therefore these coordinates cannot be considered as kinematic variables. We have found that the situation is changed if parameters of algebra $\bar{\kappa}$ are proportional to mass (18). Besides the dependence of parameters $\bar{\kappa}$ of noncommutative algebra with quadratic noncommutativity on mass is also important for description of motion of the center-of-mass of macroscopic body in quadratic space time. We have found that the Poisson brackets for coordinates and momenta of the center-of-mass do not correspond to relations of noncommutative algebra (8)-(11). This problem can be solved due to the condition (18). We have concluded that if parameters of algebra (8)-(11) corresponding to a particle are proportional to its 
mass, coordinates and momenta of the center-of-mass of a body satisfy noncommutative algebra with effective parameter $\bar{\kappa}_{e f f}$ which is proportional to the total mass of the body.

In addition we have studied the weak equivalence principle in a space with quadratic noncommutativity of coordinates. We have found that the problem of violation of this principle can be solved due to the condition (18). If relation (18) holds the trajectory of a particle (a body) do not depend on its mass and composition.

\section{Acknowledgement}

The author thanks Prof. Tkachuk V. M. for his advices and support during research studies. This work was partly supported by the Project $\Phi \Phi-63 \mathrm{Hp}$ (No. 0117U007190) from the Ministry of Education and Science of Ukraine.

\section{Список використаної літератури}

1. Witten E. Reflection on the fate spacetime / E. Witten // Phys. Today - 1996. - Vol. 49, - P. 24.

2. Doplicher S. Spacetime quantization induced by classical gravity / S. Doplicher, K. Fredenhagen, J. E. Roberts // Phys. Lett. B - 1994. - Vol. 331, No 1. - P. 39-44. doi:10.1016/0370-2693(94)909407 .

3. Djemai A. E. F. On quantum mechanics on noncommutative quantum phase space / A. E. F. Djemai, H. Smail // Commun. Theor. Phys. - 2004. - Vol. 41, No 6. - P. 837-844. doi:10.1088/0253$6102 / 41 / 6 / 837$

4. Bastos C. Probing phase-space noncommutativity through quantum mechanics and thermodynamics of free particles and quantum rotors / C. Bastos, A. E. Bernardini, J. F. G. Santos // Physica A 2015. - Vol. 438 - P. 340-354. doi:10.1016/j.physa.2015.07.009.

5. Gnatenko Kh. P. Features of free particles system motion in noncommutative phase space and conservation of the total momentum / Kh. P. Gnatenko, H. P. Laba, V. M. Tkachuk // Mod. Phys. Lett. A - 2018. - Vol. 33, No 23. - P. 1850131. doi:10.1142/S0217732318501316.

6. Gamboa J. Noncommutative quantum mechanics / J. Gamboa, M. Loewe, J. C. Rojas // Phys. Rev. D - 2001. - Vol. 64, No 6. - P. 067901. doi:10.1103/PhysRevD.64.067901

7. Romero J. M. The Kepler problem and noncommutativity / J. M. Romero, J. D. Vergara // Mod. Phys. Lett. A - 2003. - Vol. 18, No 24. - P. 1673-1680. doi:10.1142/S0217732303011472

8. Mirza B. Noncommutative geometry and classical orbits of particles in a central force potential / B. Mirza, M. Dehghani, // Commun. Theor. Phys. - 2004. - Vol. 42, No 2. - P. 183. doi:10.1088/0253$6102 / 42 / 2 / 183$

9. Daszkiewicz M. Towards quantum noncommutative -deformed field theory / M. Daszkiewicz, J. Lukierski, M. Woronowicz // Phys. Rev. D - 2008. - Vol. 77, No 10. - P. 105007. doi:10.1103/PhysRevD.77.105007

10. Daszkiewicz M. $\kappa$-deformed oscillators, the choice of star product and free $\kappa$-deformed quantum fields / M. Daszkiewicz, J. Lukierski, M. Woronowicz // J. Phys. A: Math. Theor. - 2009. - Vol. 42, No 35. - P. 355201. doi:10.1088/1751-8113/42/35/355201

11. Borowiec A. Constraints on the quantum gravity scale from $\kappa$-Minkowski spacetime / A. Borowiec Kumar S. Gupta, S. Meljanac, A. Pachol // EPL - 2010. - Vol. 92, No 2. - P. 20006. doi:10.1209/0295$5075 / 92 / 20006$

12. Borowiec A. Twisting and-Poincare / A. Borowiec, J. Lukierski, A. Pachol. // J. Phys. A: Math. Theor. - 2014. - Vol. 47, No 40. - P. 405203. doi:10.1088/1751-8113/47/40/405203

13. Borowiec A. $\kappa$-Deformations and Extended $\kappa$-Minkowski Spacetimes / A. Borowiec, A. Pachol // SIGMA - 2014. - Vol. 10 - P. 107. doi:10.3842/SIGMA.2014.107

14. Gomes M. Position-dependent noncommutativity in quantum mechanics / M. Gomes, V. G. Kupriyanov // Phys. Rev. D - 2009. - Vol. 79, No 12. - P. 125011. doi:10.1103/PhysRevD.79.125011

15. Kupriyanov V. G. A hydrogen atom on curved noncommutative space / V. G. Kupriyanov // J. Phys. A: Math. Theor. - 2013. - Vol. 46, No 24. - P. 245303. doi:10.1088/1751-8113/46/24/245303

16. Ho Pei-Ming. Noncommutative quantum mechanics from noncommutative quantum field theory/ Pei-Ming Ho, Hsien-Chung Kao // Phys. Rev. Lett. - 2002. - Vol. 88, No 2. - P. 151602. doi:10.1103/PhysRevLett.88.151602 
17. Bellucci S. Noncommutative quantum scattering in a central field/ S. Bellucci, A. Yeranyan // Phys. Lett. B- 2005. - Vol. 609, No 3-4. - P. 418. doi:10.1016/j.physletb.2005.01.058

18. Daszkiewicz M. Classical mechanics of many particles defined on canonically deformed nonrelativistic spacetime/ M. Daszkiewicz, C. J. Walczyk // Mod. Phys. Lett. A - 2011. - Vol. 26, No 11. - P. 819-832. doi:10.1142/S0217732311035328

19. Daszkiewicz M. Quantum mechanics of many particles defined on twisted N-enlarged NewtonHooke space-times/ M. Daszkiewicz // Acta Phys. Polon. B - 2013. - Vol. 44, No 4. - P. 699-711. doi:10.5506/APhysPolB.44.699

20. Gnatenko Kh. P. Weak equivalence principle in noncommutative phase space and the parameters of noncommutativity / Kh. P. Gnatenko, V. M. Tkachuk // Phys. Lett. A - 2017. - Vol. 31, No 31. P. 2463-2469. doi:10.1142/S0217732317501668

21. Gnatenko Kh. P. Composite system in noncommutative space and the equivalence principle / Kh. P. Gnatenko // Phys. Lett. A - 2013. - Vol. 377, No 43. - P. 3061-3066. doi:10.1016/j.physleta.2013.09.036

22. Gnatenko Kh. P. Kinematic variables in noncommutative phase space and parameters of noncommutativity / Kh. P. Gnatenko // Mod. Phys. Lett. A - 2017. - Vol. 32, No 31. - P. 1750166. doi:10.1142/S0217732317501668

23. Gnatenko Kh. P. Rotationally invariant noncommutative phase space of canonical type with recovered weak equivalence principle / Kh. P. Gnatenko // EPL - 2018. - Vol. 123, No 5. - P. 50002. doi:10.1209/0295-5075/123/50002

24. Tkachuk V. M. Deformed Heisenberg algebra with minimal length and the equivalence principle / V. M. Tkachuk // Phys. Rev. A - 2012. - Vol. 86, No 6. - P. 062112. doi:10.1103/PhysRevA.86.062112

25. Tkachuk V. M. Galilean and Lorentz transformations in a space with Generalized Uncertainty Principle / V. M. Tkachuk // Found. Phys. - 2016. - Vol. 46, No 12. - P. 1666-1679. doi:10.1007/s10701016-0036-5

26. Daszkiewicz M. Newton equation for canonical, Lie-algebraic, and quadratic deformation of classical space / M. Daszkiewicz, C. J. Walczyk // Phys. Rev. D - 2008. - Vol. 77, No 10. - P. 105008. doi:10.1103/PhysRevD.77.105008

\section{References}

1. E. Witten, Phys. Today. 49, 24 (1996).

2. S. Doplicher, K. Fredenhagen and J. E. Roberts, Phys. Lett. B 331, 39-44 (1994). doi:10.1016/03702693(94)90940-7.

3. A. E. F. Djemai and H. Smail, Commun. Theor. Phys. 41, 837-844 (2004). doi:10.1088/0253$6102 / 41 / 6 / 837$.

4. C. Bastos, A. E. Bernardini and J. F. G. Santos, Physica A. 438, 340-354 (2015) doi:10.1016/j.physa.2015.07.009.

5. Kh. P. Gnatenko, H. P. Laba and V. M. Tkachuk, Mod. Phys. Lett. A 33, 1850131 (2018). doi:10.1142/S0217732318501316.

6. J. Gamboa, M. Loewe and J. C. Rojas, Phys. Rev. D 64, 067901 (2001) doi:10.1103/PhysRevD.64.067901

7. J. M. Romero and J. D. Vergara, Mod. Phys. Lett. A , 1673-1680 (2003). doi:10.1142/S0217732303011472

8. B. Mirza and M. Dehghani,, Commun. Theor. Phys. 42, 183 (2004). doi:10.1088/0253-6102/42/2/183

9. M. Daszkiewicz, J. Lukierski and M. Woronowicz, Phys. Rev. D 77, 105007 (2008). doi:10.1103/PhysRevD.77.105007

10. M. Daszkiewicz, J. Lukierski and M. Woronowicz, J. Phys. A: Math. Theor. 42, 355201 (2009). doi:10.1088/1751-8113/42/35/355201

11. A. Borowiec, Kumar S. Gupta, S. Meljanac and A. Pachol, EPL 92, 20006 (2010). doi:10.1209/0295$5075 / 92 / 20006$

12. A. Borowiec, J. Lukierski and A. Pachol., J. Phys. A: Math. Theor. 47, 405203 (2014). doi:10.1088/1751-8113/47/40/405203

13. A. Borowiec and A. Pachol, SIGMA 10, 107 (2014). doi:10.3842/SIGMA.2014.107

14. M. Gomes and V. G. Kupriyanov, Phys. Rev. D 79, 125011 (2009). doi:10.1103/PhysRevD.79.125011

15. V. G. Kupriyanov and J. Phys. A: Math. Theor. 46, 245303 (2013). doi:10.1088/1751$8113 / 46 / 24 / 245303$ 
16. Pei-Ming Ho and Hsien-Chung Kao, Phys. Rev. Lett. 88, $88151602 \quad$ (2002). doi:10.1103/PhysRevLett.88.151602

17. S. Bellucci and A. Yeranyan, Phys. Lett. B 609, 418 (2005). doi:10.1016/j.physletb.2005.01.058

18. M. Daszkiewicz and C. J. Walczyk, Mod. Phys. Lett. A 26, $819-832$ (2011). doi:10.1142/S0217732311035328

19. M. Daszkiewicz, Acta Phys. Polon. B 44, 699-711 (2013). doi:10.5506/APhysPolB.44.699

20. Kh. P. Gnatenko and V. M. Tkachuk, Phys. Lett. A 31, 2463-2469 (2017). doi:10.1142/S0217732317501668

21. Kh. P. Gnatenko, Phys. Lett. A 377, 3061-3066 (2013). doi:10.1016/j.physleta.2013.09.036

22. Kh. P. Gnatenko, Mod. Phys. Lett. A 32, 1750166 (2017). doi:10.1142/S0217732317501668

23. Kh. P. Gnatenko, EPL 123, 50002 (2018). doi:10.1209/0295-5075/123/50002

24. V. M. Tkachuk, Phys. Rev. A 86, 062112 (2012). doi:10.1103/PhysRevA.86.062112

25. V. M. Tkachuk, Found. Phys. 46, 1666-1679 (2016). doi:10.1007/s10701-016-0036-5

26. M. Daszkiewicz and C. J. Walczyk, Phys. Rev. D 77, 77105008 (2008). doi:10.1103/PhysRevD.77.105008 


\section{Зв'язок параметрів некомутативної алгебри з масою та принцип еквівалентності у просторі з квадратичною некомутативністю координат}

\section{Х. П. Гнатенко, О. I. Григорчак}

Лъвівсъкий націоналъний університет імені Івана Франка, Кафедра теоретичної фізики вул. Драгоманова, 12, 79005 Львів, Україна e-mail: khrystyna.gnatenko@gmail.com

Розглянено квантований простір, який описується алгеброю з квадратичною некомутативністю координат. Досліджується загальний випадок, коли рух різних частинок у квантованому просторі описується різними параметрами некомутативності. Ми виявили, що ідея залежності параметрів некомутативної алгебри від маси дозволяє отримати ряд важливих результатів у просторі з квадратичною некомутативністю координат. А саме, ми показали, що у випадку, коли параметр некомутативності є прямопропорційним до маси, координати у некомутативному просторі не залежать від маси та можуть розглядатися як кінематичні змінні. Також зв'язок параметра некомутативності з масою є важливим для опису руху системи багатьох частинок у просторі з квадратичною некомутативністю. А саме, ми знайшли, що дужки Пуассона для координат та імпульсів центра мас відповідають співвідношенням некомутативної алгебри з ефективним параметром некомутативності, якщо відношення параметра некомутативності до маси є сталим для різних частинок. Варто зазначити, що ефективний параметр некомутативності визначається повною масою системи та не залежить від іiі композиції. Також важливо відзначити, що вплив некомутативності на рух центра мас системи частинок зменшується при збільшенні маси системи. Макроскопічні тіла відчувають менший вплив квантованості простору у порівнянні з елементарними частинками. На додаток, у випадку прямопропорційної залежності параметрів некомутативної алгебри від маси рух

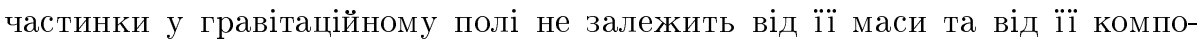
зиції. Отже, відновлюється слабкий принцип еквівалентності у просторі з квадратичною некомутативністю координат. Важливо зазначити, що ідея залежності параметрів алгебри, яка описує квантований простір, від маси дозволяє розв'язати важливі проблеми також у некомутативному фазовому просторі канонінчного типу, у деформованому просторі з мінімальною довжиною.

Key words: квадратична некомутативність, принцип еквівалентності, кінематичні змінні 


\section{Связь параметров некоммутативной алгебры с массой и принцип эквивалентности в пространстве с квадратичной некомутативностю координат}

\section{Х. П. Гнатенко, О. И. Григорчак}

Лъвовский национальный университет имени Ивана Франко, Кафедра теоретической физики ул. Драгоманова 12, 79005 Львов, Украина e-mail: khrystyna.gnatenko@gmail.com

Найдено, что соотношение параметров некоммутативной алгебры с массой дает возможность решать важные задачи в квадратичном пространствевремени. Мы показываем, что если параметр некоммутативноий алгебры пропорционален массе, то координаты в квадратичном пространствевремени можно рассматривать как кинематические переменные, также восстанавливается слабый принцип эквивалентности и соотношения для координат и импульсов центра масс тела соответсвуют соотношениям некоммутативной алгебры с эффективным параметром.

Ключевые слова: квадратичная некомутативность, принцип эквивалентности, кинематические переменные

Статтю отримано: 12.06.2019

Прийнято до друку: 24.06.2019 